\section{Esclerosis Múltiple: Una Mirada Ibero-Panamericana, 2008}

$\mathrm{H}$ e aceptado la gentil invitación del Editor de la Revista para presentar la segunda edición del libro de C. Arriagada y J. Nogales-Gaete (Editores) Esclerosis Múltiple: Una Mirada IberoPanamericana, New York, Demos Medical Publishing 2008.

La sola concreción de una segunda edición de este libro nos llena alegría. Para esta segunda oportunidad hemos logrado aumentar la participación de destacados autores nacionales y extranjeros de 48 a 128, ampliando y actualizando significativamente el arco temático de 33 a 70 capítulos. Por otro lado, hemos tenido el respaldo formal de dos importantes referentes multinacionales del tema, como son LACTRIMS: Comité Latinoamericano para la Investigación y Tratamiento de la Esclerosis Múltiple y CMSC: Consortium of Multiple Sclerosis Centres. La edición ha sido realizada por la prestigiosa editorial Demos Medical Publishing de Nueva York. Hemos realizado dos lanzamientos internacionales del libro en el marco del congreso 2008 del CMSC en Denver USA y del Primer Congreso Mundial de Esclerosis Múltiple en Montreal Canadá.

Todos estos hitos dan cuenta de un implícito reconocimiento a un tipo de convocatoria y trabajo sistemático, que se ha mantenido por años constituyéndose en la práctica en una escuela formadora iniciada por el Prof. Dr. Camilo Arriagada, de la que estoy orgulloso de ser parte.

Como justificación especial para mantener este esfuerzo editorial latinoamericano, en un área de tantas y calificadas publicaciones clásicas y emergentes, considero que debemos admitir que se trata de una tarea simplemente ineludible. Existen dominios del conocimiento donde nuestra información y experiencias como latinoamericanos son insustituibles: la epidemiología, el diagnostico diferencial, la relación médico-pacientes, las percepciones de los pacientes, la respuesta a tratamiento, entre otros, tienen relevantes particu- laridades regionales genéticas sociales, culturales y sanitarias. Esto determina que la información originada en otras realidades, independiente de lo calificado del autor o centro de origen, no pueda reemplazar nuestros aportes, entregándonos la oportunidad y desafío de esta tarea.

Para presentar el libro en comento recurriré a algunas citas vertidas como prólogos del mismo libro por destacadas autoridades que trabajan en investigación, enseñanza y práctica clínica relacionada con Esclerosis Múltiple (EM).

Dr. Camilo Arriagada, Maestro de la Neurología de Sonepsyn, Profesor Titular de Neurología Universidad de Chile, Staff del Servicio de Neurología y Neurocirugía del Hospital del Trabajador y coeditor del libro.

Es una obra maciza, moderna, actualizada y con muchos aportes originales, incluyendo además contribuciones de eminencias como Kurtzke, Poser, y Fernández que le darán especial trascendencia en el tiempo ya que constituirán de seguro un hito histórico.

Dr. Alan J. Thompson, Editor of Multiple Sclerosis Chairman of the Medical and Scientific Board of International Federation of Multiple Sclerosis Societies. Professor of Clinical Neurology and Neurorehabilitation, Institute of Neurology, University of London UK.

Este exhaustivo texto que presento, aporta importantes contribuciones, muchas de ellas originadas en toda la larga y ancha extensión de América del Sur. Está escrito por expertos locales en la materia, y provee una maravillosa oportunidad para sus lectores al entregar información que permitirá elevar el perfil de conocimiento, alerta y sensibilidad sobre la EM, a la vez de estimular la búsqueda de los mejores estándares en el cuidado de los pacientes con EM.

Es un gran placer para mí como Chairman of the Medical and Scientific Board of the Multiple Sclerosis International Federation, recomendar este libro y estimular a los lectores a perseverar en la continua mejoría del trabajo de asistir a quienes están afectados por la EM. 
Dr. Víctor M. Rivera, Presidente LACTRIMS 2001-2005, Profesor de Neurología y Director, del Multiple Sclerosis Center Baylor College of Medicine Texas Medical Center de Houston, USA.

Esclerosis Múltiple: Una Mirada Ibero-Panamericana se constituye en una importante obra sobre la Esclerosis Múltiple escrita en castellano. Esta histórica producción cubre desde la comprensión básica de mecanismos de inmunopatogenia, destrucción oligodendroglial, disfunción axonal, explora los misterios de la barrera hematoencefálica y discute la EAE como modelo animal. La segunda edición de este texto magnificado incluye diversos apuntes $y$ datos epidemiológicos ya sea por recolección global $o$ por estudios regionales, junto a comentarios sobre asistencia sanitaria. La obra dedica grandes segmentos a los aspectos y variedades clínicas, neuromielitis óptica, encefalomielitis, los criterios nuevos para diagnóstico (incluyendo el diferencial y discusión de algunos de ellos), EM pediátrica, los sintomas, los tratamientos básicos actuales y los visualizados en el horizonte.

Esta nueva mirada incluye detalles de gran significado para la posteridad como la inusual y extraordinaria contribución de padres intelectuales del estudio contemporáneo de la EM como los profesores Kurtzke y Poser y así mismo la participación de otros líderes de opinión internacional y otras personas distinguidas por su perenne batallar por siempre para mejorar la atención y el cuidado de los afectados por la enfermedad.

La lista de autores (128 en 70 capitulos) incluye colegas de Argentina, Bolivia, Brasil, Canadá, Chile, Colombia, Croacia, Cuba, Ecuador, España, Estados Unidos, México, Panamá, Paraguay, Perú, Portugal, Reino Unido, Uruguay y Venezuela. Apreciamos la obra de Camilo Arriagada y Jorge Nogales-Gaete, editores de Esclerosis Múltiple: Una Mirada Ibero-Panamericana quienes en este texto de conjunción multinacional y original (primero en su especie en Latinoamérica y posiblemente en el mundo) le ofrecen a una audiencia multidisciplinaria ávida de información, un positivo aporte para la educación integral sobre la EM.
Carlos Oehninger Gatti, Presidente LACTRIMS 2007-2009, Profesor de Clínica Neurológica Instituto de Neurología, Facultad de Medicina de la Universidad de la República Oriental del Uruguay.

Esta obra es de gran valor por la temática abarcada, que va desde los aspectos básicos hasta las etapas diagnósticas, clínicas y paraclinicas, pasando por la epidemiología general y de cada país, de niveles de asistencia en distintos sectores de Latinoamérica, por las manifestaciones clínicas tan variables, las diferentes formas clínicas posibles, la evaluación de la discapacidad que ella conlleva, los sindromes restrictos, los trastornos neurocognitivos y los diagnósticos diferenciales más probables. En base a un gran esfuerzo, los editores incorporan en esta 2a edición de "Esclerosis Múltiple. Una Mirada Ibero- Panamericana" aspectos novedosos de tratamiento, y aspectos de excepcional importancia, tanto para la persona que padece la enfermedad como para el neurólogo de cabecera, en relación a la información del diagnóstico nosológico y a la importancia que tienen en la lucha contra la enfermedad, las asociaciones de pacientes.

El valor didáctico y de aplicación práctica de esta obra será para el neurólogo clínico un instrumento verdaderamente esencial para el diagnóstico, tratamiento y manejo de la persona con EM. Lo será también para el conjunto de especialistas que integran el equipo interdisciplinario necesario para el tratamiento actual de esta patología y redundará sin lugar a dudas en una notable mejoría de la calidad de vida de las personas con EM.

Dr. Marco Aurélio Lana-Peixoto, Presidente LACTRIMS 2005-2007. Profesor de Neurología y Oftalmología, Universidade Federal de Minas Gerais, Belo Horizonte, Brasil.

Este libro permite no sólo el acceso a calificados textos originales en español, sino a toda una reflexión y experiencia, que da cuenta dirigidamente de las particularidades socio-culturales, asistenciales, genéticas y ambientales de la región.

Gracias a los infatigables y destacados esfuerzos personales de los editores, Camilo Arriagada y Jorge Nogales-Gaete, y a su reconocido liderazgo, se 
ha podido congregar en esta obra la valiosa contribución de destacados médicos y otros profesionales que trabajan en el tema de la EM, no sólo en América Latina, sino en España, EEUU, Canadá, Portugal y Croacia, generando un instrumento actualizado de inestimable valor educacional y clínico, que de seguro ayudará a comprender mejor la EM, para asistir mejor a quienes la padecen, en nuestra región.

Sólo nos resta invitarles a considerar esta obra al momento de requerir una alternativa en el tema. Si bien no esta disponible físicamente en el mercado nacional, es posible obtenerla a través de Internet en múltiples librerías on-line de diversos países: España, México, USA, Canadá, Japón, Corea, Finlandia, Holanda, Reino Unido, Noruega, Rusia, Dinamarca y Alemania. Para contactar una de estas librerias basta ir a Google y buscar: "Esclerosis Múltiple: Una Mirada Ibero-Panamericana" o ir a la tienda de Demos Medical Publishing (www.demosmedpub.com).

\footnotetext{
Jorge Nogales-Gaete jnogales@ctcinternet.cl
} 